\title{
Habiter WhatsApp ? Éléments d'analyse postdualiste des interactions en espace numérique
}

Yosra Ghliss

Université Paul-Valéry Montpellier 3, Praxiling UMR 5267-CNRS

yosra.ghliss17@gmail.com

Marc Jahjah

Université de Nantes, laboratoire LS2N

marc.jahjah@univ-nantes.fr

\section{Introduction}

C'est d'abord chez les sociologues que l'hypothèse a germé : en menant une série d'entretiens sur WhatsApp, Kenton O'hara et ses collègues (2014) ont constaté que ses utilisateurs concevaient l'application comme un espace alternatif dans lequel se pratiquent les activités de la vie quotidienne : papoter, s'entraider, partager des anecdotes, des photos, etc. Dans cette même étude, ils ont affiné la notion de l'«habiter» (dwelling) de Tim Ingold (2013). Nous nous proposons de vérifier cette hypothèse en adoptant le prisme épistémologique de la linguistique. À partir d'une observation empirique d'un corpus WhatsApp, une nouvelle conception de l'espace semble émerger. L'espace numérique n'est plus un simple paramètre que les locuteurs actualisent dans le discours, encore moins un «extérieur » auquel ils se réfèrent; il est constitutif de l'activité interactionnelle. Nous proposons de vérifier cette hypothèse en développant un cadre théorique qui puise dans différentes disciplines articulées aux sciences du langage. Nous en vérifions ensuite la pertinence sur un corpus d'historiques WhatsApp avant de proposer une typologie de l'activité interactionnelle dans cet espace numérique.

\section{Cadrage théorique : l'habitat comme modalité de l'espace numérique}

La notion de «l'habiter» n'est pas une notion linguistique. Elle semble pourtant pertinente et fertile pour penser les espaces numériques. Dans cette partie, nous proposons ainsi une exploration théorique dont nous tirerons les leçons pour les sciences du langage et particulièrement l'analyse de discours. L'analyse du discours numérique (Paveau 2017), à laquelle nous souscrivons méthodologiquement, permet certes de travailler les énoncés numériques. Mais l'appropriation et la création d'un espace interrelationnel, qui nous intéressent aussi ici, imposent de résoudre quelques problèmes : quand et comment habitons-nous un espace? Qu'est-ce qu'un «monde à soi »? Ces questions sont au cœur des préoccupations des philosophes et sociologues contemporains de l'espace (Paquot et al. 2007), ainsi que des anthropologues et des phénoménologues et plus généralement des chercheurs en sciences humaines et sociales (Agostini-Marchese 2017). Nous suggérons de mobiliser les travaux des disciplines comme la philosophie et l'anthropologie pour en examiner les résonances.

\section{1. Habiter l'espace : objet d'étude interdisciplinaire}

\section{1. 1. La «fonction d'habiter » en philosophie}

On trouve chez Gaston Bachelard des premières réponses sérieuses sur la question de l'habiter, dans la lignée de la phénoménologie de l'espace vécu des années 1960 (Herouard 2007). En sortant d'une compréhension strictement structurale et géographique de l'espace, sa poétique (1957) se livre à une analyse topographique de notre intimité en révélant les lieux où elles se nichent (le coin, le tiroir, l'armoire, la maison, etc.) et les formes qu'elle prend (le nid, la spirale, le rond). Pour Bachelard, l'espace habité est un espace qui révèle une organisation et un agencement singuliers, nourris par un 
imaginaire archétypal. Ainsi, la cave "participe aux puissances souterraines », tandis que la maison est force de rassemblement : elle concentre les souvenirs de ses occupants, constamment menacés par la dispersion. La «fonction d'habiter » consiste ainsi à faire d'un espace donné un espace plus ou moins personnel par le truchement d'imaginaires et de verbes d'action (se loger, se couvrir, se protéger, etc.) qui le travaillent dans son organisation. C'est pourquoi Bachelard écrit : « [u]n être vivant emplit un refuge vide»(1957:133). Nous avons la capacité d'occuper un espace en y projetant notre vie intime, constituée de nos affects, de nos souvenirs, de notre relation aux autres. Pour le dire autrement : nous nous déposons dans les objets qui nous entourent.

Dans cette «topo-analyse », les coins et les nids font l'objet d'une attention particulière : ils incarnent les formes élémentaires de la «fonction d'habiter », qui revient à se blottir, à définir un espace à soi dans un monde beaucoup trop vaste pour soi (car $«$ dans l'infini on n'est pas chez soi $\left.{ }^{1}\right)$. Ainsi, les petits espaces, comme les coins et les nids, sont-ils métonymiques (ils concentrent tout un monde) et précaires : s'ils offrent un réconfort, ils sont menacés par le monde où ils prennent forme, tout comme les êtres vivants qui les occupent. Les êtres et les espaces sont étroitement liés : nous sommes nos espaces.

\section{1. 2. Approche anthropologique de l'environnement}

L'anthropologie écologique de Ingold (2011) adopte un point de vue quelque peu similaire : l'espace habité est mon environnement, c'est-à-dire « le monde tel qu'il existe et acquiert une signification par rapport à moi ». (2013 : 18) Les espaces sont pensés de manière dynamique : loin d'être inertes, ils sont en perpétuelle élaboration grâce aux activités des êtres vivants qui les habitent, les pensent, découvrent leur signification en fonction de leurs intérêts propres. L'anthropologie écologique décrit un mouvement d'oscillation permanent et constitutif entre les êtres et leurs environnements, qui sont éminemment vivants. La notion d' «affordance », empruntée à James J. Gibson (1977), joue un rôle majeur dans cette co-constitution. Dans la perspective de Ingold, elle désigne l'ensemble des propriétés réelles qui rendent un objet susceptible de prendre part au projet d'un sujet. L'intentionnalisme, identifié par les phénoménologues, guide ainsi notre capacité à saisir dans notre environnement des prises (ou fonctions) qui nous permettent de résoudre une problématique donnée. Autrement dit: nous ne percevons que ce dont nous avons besoin pour orienter notre action. Pour autant, les éléments non perçus existent objectivement, indépendamment de nous : ils attendent d'être découverts ou actualisés grâce au projet dont se dote un individu. Si les espaces sont déjà signifiants, avant même qu'un individu ne s'en empare, s'il ne fait que redécouvrir leur signification, c'est parce que ces espaces s'inscrivent dans une histoire: ils ont été travaillés par des humains et des nonhumains ; ils sont déjà sémiotisés. Nous n'arrivons pas vierges pour doter le monde de significations en y plaquant nos grilles culturelles : il ne nous a pas attendus pour exister. Notre action est modeste mais essentielle : elle consiste à inscrire le monde dans un projet personnel en infléchissant un temps sa course et son flux. C'est ce que Ingold appelle «environnement» et André Leroi-Gourhan le «rythme» (1945): nous sélectionnons dans notre espace les éléments pertinents qui nous aident à réaliser une tâche donnée et à répondre aux problèmes, défis, enjeux du monde par l'invention de prises continuelles, comme des calendriers pour se souvenir ou des concepts pour penser. L'environnement, comme le rythme, est un mouvement continu.

\section{1. 3. Les « régimes d'habiter»}

\footnotetext{
${ }^{1}$ Bachelard, interrogé par Paule Chavasse en 1959 sur les ondes de la Radio Diffusion Française à propos de son essai La Poétique de l'espace. Émission consultable en ligne : <https://www.franceculture.fr/emissions/lesnuits-de-france-culture/interview-de-gaston-bachelard-1ere-diffusion-13021963>, consultée le 01/10/2018.
} 
Les théories modernes de l'habiter convergent vers la même idée (Stock 2007) : l'espace est une ressource pour l'action; il offre des prises. Les individus sont à la fois de l'espace mais font également avec de l'espace qui ne prédéfinit jamais leur course, même s'il a déjà été façonné par d'autres mains et d'autres projets. Ainsi, l'habiter est « polytopique» (Stock $2007: 111$ ) : l'espace se modifie selon les intentions et pratiques des êtres vivants. Habiter, c'est pratiquer l'espace, à la fois ressource de l'action et condition de cette action : il se constitue comme environnement à mesure qu'il est découvert et travaillé par les situations personnelles des individus qui mobilisent des compétences déictiques («gestions de l'ici, de l'ailleurs»), spatiales (ils font littéralement avec les contraintes des lieux) et expérientielles (les lieux sont des épreuves : ils résistent à nos projets, nous obligent à franchir des obstacles). Or, ces pratiques s'inscrivent dans un ordre : elles doivent à la fois obéir à des contraintes matérielles et sociales; elles ne sont jamais que potentielles car elles doivent faire la démonstration de leur insertion. Elles sont toujours comprises dans un « régime d'habiter ».

\section{2. Éléments pour penser l'espace numérique}

\subsection{Le numérique : espace discursif complexe}

Comment l'espace a-t-il été pensé, dans un contexte numérique ? Depuis une dizaine d'années déjà, Milad Doueihi attire notre attention sur l'espace habitable que crée le code informatique (2011) : en s'ajustant sans cesse aux pratiques culturelles, qu'il ajuste parallèlement à ses propres prérogatives, il favorise l'émergence d'une culture numérique. Cette dernière se caractérise par une hybridité généralisée des espaces de la vie quotidienne, à tel point qu'il n'est plus possible de distinguer un espace en ligne et hors ligne : nous sommes continuellement traversés de part et d'autre par le code informatique, qu'il s'agisse de se repérer dans la ville, d'organiser un événement via un réseau (dit) social ou d'ouvrir la porte d'un magasin (Kitchin \& Dodge 2011). Le corps humain est ainsi constamment appareillé par un corps technique qui lui offre des prises et redéfinit ses propres techniques corporelles, pour reprendre une formule de Marcel Mauss². Nous sommes passés d'une terre habitée à une spatialité élargie. À la suite de Leroi-Gourhan, Doueihi pense ainsi l'espace comme un milieu, c'est-à-dire une articulation entre des gestes, des matériaux, des signes, des objets, des sites qui définissent les lieux de vie des hommes, tendus entre continuité et discontinuité.

On trouve dans la théorie de l'éditorialisation ${ }^{3}$ une pensée semblable (Agostini-Marchese 2017) : elle reconnaît à l'individu la capacité de construire son propre espace dans le flux des signes, des matériaux, des dispositifs qui s'offrent à lui et le contraignent conjointement. Un tel travail relève de l'anamorphose : il consiste à se saisir de toutes les prises du monde pour construire un espace hybridé, gradué, intime, qui allie des instances variées, aussi bien numériques que non numériques. Cette distinction ne tient plus dans la théorie de l'éditorialisation: comme chez Doueihi, nous sommes constamment traversés par les objets techniques, sans qu'il soit possible de déterminer quelle part revient aux uns et aux autres. L'espace est un agencement de pratiques, d'objets, d'hommes, de dispositifs, de signes.

\section{2. 2. Hypothèse : habiter l'espace numérique ?}

\footnotetext{
${ }^{2}$ Le concept de «techniques du corps » sert à désigner la nature sociale des attitudes, gestes, postures corporelles. Ce sont des créations humaines qui varient historiquement et spatialement; nous les apprenons, les incorporerons, grâce à un processus de socialisation.

${ }^{3}$ Cette théorie est élaborée depuis quelques années par Marcelo Vitali-Roseti et son groupe de recherche (Chaire des écritures numériques de l'université de Montréal). L'éditorialisation cherche à qualifier les propriétés de l'Internet et du web en les saisissant à l'intersection de la spatialité, de la technique et des pratiques.
} 
Comment tirerons-nous parti de tous ces éléments pour travailler notre propre corpus d'historiques de conversation de WhatsApp ? Avant de proposer des premières pistes d'articulation avec les sciences du langage, résumons les temps forts des travaux évoqués : certes, l'homme habite le monde en y projetant ses propres imaginaires pour le rendre plus familier, le domestiquer, y définir des coins dans lesquels se blottir et s'étendre progressivement pour faire monde (Bachelard, 1957). Mais le monde n'est pas vierge pour autant : il existe indépendamment de nous-mêmes, il a déjà été constitué par d'autres, pour d'autres ; nous ne faisons que découvrir ses localités à partir des projets et des besoins que nous avons (Ingold). Ainsi, habiter le monde est une activité polytopique : l'espace se redéfinit sans cesse à partir des pratiques déictiques, spatiales et expérientielles des individus qui éprouvent les lieux, font avec leurs contraintes, les détournent. L'espace numérique est soumis aux mêmes problématiques : il n'est pas donné, pas même dans une interface figée ; il est toujours coconstruit par les individus ; c'est un milieu en perpétuelle redéfinition qui retravaille à la fois les espaces de vie, nos habitus et nos techniques corporelles.

Dans les sciences du langage, nous trouvons des échos à nos réflexions et des appuis épistémologiques dans les travaux de deux chercheuses. D'un côté, Lorenza Mondada en linguistique interactionnelle $(2005,2012)$, a investi l'approche praxéologique pour examiner la référence à la spatialité mais aussi la gestion, et l'implication de l'espace dans l'activité interactionnelle. De l'autre, Marie-Anne Paveau en analyse du discours $(2012,2017)$ propose une approche symétrique l'espace numérique. En d'autres termes, elle rompt avec l'opposition des activités «hors ligne» et des activités «en ligne » (2017) mais invite au contraire à les appréhender en continuum puisque tous deux participent à la constitution d'un milieu commun, par le truchement des activités technolangagières. De fait, l'espace numérique est bien dynamique : en recourant aux affordances (hashtags, émoticons, etc.) de leurs environnements, les locuteurs construisent des localités, des zones énonciatives et langagières qui leur sont propres. D'où le recours aux analyses qualitatives pour en rendre compte, dans une perspective écologique, qui tienne compte de l'environnement iconique, technique, verbal des énoncés.

Nous nous demanderons ainsi comment les individus tirent parti de leur environnement technicolangagier pour construire un espace intersubjectif et relationnel : où se situent leurs petits mondes à eux, dans WhatsApp ? Sur quelles affordances s'appuient-ils pour les fabriquer ? Peut-on identifier une continuité entre WhatsApp et les espaces tangibles des individus ? Quelles pratiques déictiques, spatiales et expérientielles mobilisent-ils pour habiter WhatsApp ?

\section{L'application WhatsApp : présentation du corpus, description de l'interface numérique}

\section{1. Corpus d'étude}

Notre article s'appuie sur un corpus d'interactions de l'application WhatsApp, disponible sur plusieurs systèmes d'exploitation (Android, iOS) et supports (téléphones, tablettes). WhatsApp est une application logicielle de messagerie instantanée via Internet. À ce jour, il s'agit de l'application de messagerie la plus utilisée à l'échelle mondiale comptant à son actif plus de 1,5 milliard d'utilisateurs actifs dans le monde (chiffre enregistré en août 2018) ${ }^{4}$. Sa plateforme multimodale permet de converser à deux ou en groupe. Par ailleurs, les interactions qui y sont accomplies peuvent être écrites, iconiques, audio ou encore vidéo (voir 2.2). Cette particularité n'est donc pas sans conséquence quant à la nature des données collectées qui peuvent sembler a priori hétéroclites. Le corpus dont nous

\footnotetext{
${ }^{4}$ Voir en ligne : <http://www.alexitauzin.com/2013/04/combien-dutilisateurs-de-facebook.html $>$, consulté 02/10/2018.
} 
disposons regroupe un ensemble de conversations écrites mais intègre aussi des photos et les enregistrements audio qui ont été échangés lors de ces discussions. Nous ne disposons pas d'interactions par vidéo ou même entièrement audio puisque WhatsApp propose de sauvegarder uniquement les conversations écrites avec les fichiers (audio, photos, autre documents) échangés. Trois particularités de notre corpus méritent d'être soulignées : 1) sa taille ;2) la nature des échanges ; 3) le caractère sensible des données :

(1) S'inscrivant dans une démarche qualitative du discours, le corpus nous est principalement utile pour observer, catégoriser et enfin analyser en contexte quelques extraits. Il n'a pas pour vocation la représentativité. Il s'agit donc d'un "petit corpus ${ }^{5}$ constitué de 34 historiques provenant de 15 participants francophones ayant accepté d'envoyer leurs historiques de conversation WhatsApp dans le cadre d'une recherche en cours (Ghliss à paraître en 2019). Chaque participant a envoyé entre 1 et 6 historiques.

(2) Les échanges dont nous disposons sont spontanés et authentiques. En d'autres termes, notre démarche consistait à solliciter le public pour nous envoyer des historiques de conversation, conversations déjà tenues avec leur entourage. Les échanges ont donc été menés dans leur cadre ordinaire naturel et la collecte s'est faite en aval. Autrement dit, ces échanges n'ont été ni fabriqués ni suscités et n'ont pas eu lieu en présence des chercheurs. Les conversations de notre corpus renvoient à des activités interactionnelles variées : allant d'une discussion entre deux copines pendant deux ans à un échange succinct entre deux collègues en passant par des conversations entre membres d'une même famille. Ce qui nous emmène à notre troisième point : la nature des données collectées.

(3) Les échanges WhatsApp relèvent de la donnée privée. Juridiquement, ils ne sont pas du même ordre qu'un tweet public ou un statut Facebook. Ces conversations sont des échanges contenant des données privées qui doivent être masquées pour préserver l'identité des participants. Nous avons ainsi procédé à l'anonymisation des données personnelles en suivant les recommandations de la CNIL. Ainsi, toute indication permettant de reconnaître un tiers a été modifiée (nom, prénom, etc. pour les conversations écrites) ou floutée (pour les photos).

\section{2. Description de l'interface}

Afin de mieux comprendre ces données, nous proposons d'examiner au préalable leur environnement numérique. La lecture d'un point de vue sémiotique de l'interface de WhatsApp permet de saisir la dynamique visuelle et l'architecture dans laquelle les interactions ont été conçues, perçues mais aussi vécues. L'écologie du discours « repose sur l'idée que les discours sont constitutivement intégrés à leurs contextes, et qu'ils ne peuvent être analysés à partir de leur seule matière langagière, mais comme composites métissant de manière intrinsèque du langagier et du technologique, etc.» (Paveau 2017: 203). À partir de ce point de vue, la phase de description d'interface semble essentielle, car elle invite à prendre en compte par la suite dans l'analyse, non seulement le contexte (entendu comme cadre sociohistorique) mais aussi l'environnement matériel de ces énoncés. Il s'agit en quelque sorte de restituer la matière langagière et discursive collectée en corpus dans son environnement technologique :

Regardée à partir d'une perspective linguistique, l'étude montre que les discours sont contextuels : nous produisons nos discours, non à partir d'une intentionnalité hors-sol ni

\footnotetext{
${ }^{5}$ Voir le numéro « Les petits corpus », Corpus 18, mis en ligne le 03/07/2018, consulté le 08/03/2019. En ligne : $<$ http://journals.openedition.org/corpus/3099>.
} 
d'une subjectivité à l'illusoire autonomie, mais au sein d'un environnement qui nous donne des instructions sémantiques fortes. (Paveau 2017 : 25)

C'est donc à partir de cet environnement qui donne des «instructions sémantiques fortes », comme toute affordance, que les locuteurs interagissent, et c'est ce même environnement que nous entendons analyser dans un premier temps avant d'examiner les échanges qui y sont produits. WhatsApp est en effet une application multiplateforme (dans la mesure où elle propose différents types d'échanges écrits, audio et vidéo) qui propose un dispositif communicationnel régi par un ensemble d'affordances rendant possibles des conversations multimodales et plurisémiotiques (Ghliss à paraître en 2019).

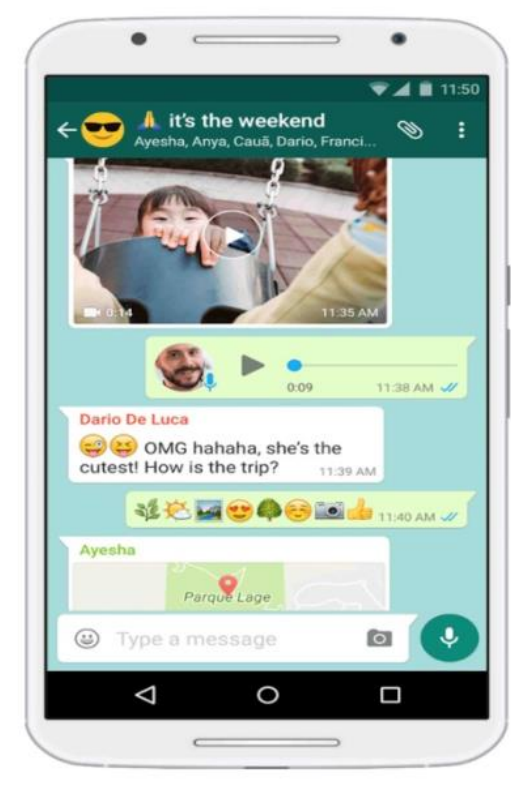

Illustration $\mathrm{n}^{\circ} 1$ : Photo extraite du site officiel de WhatsApp ${ }^{6}$

Le dispositif WhatsApp donne la possibilité de créer des fils de discussion à deux ou en groupe. L'application propose à l'usager la liste de ses contacts qui sont sur WhatsApp créant ainsi des espaces de discussion à l'image de pièces vides (voir illustration $\mathrm{n}^{\circ} 2$ ).

${ }^{6}$ Voir en ligne $:<$ https://www.whatsapp.com>. 


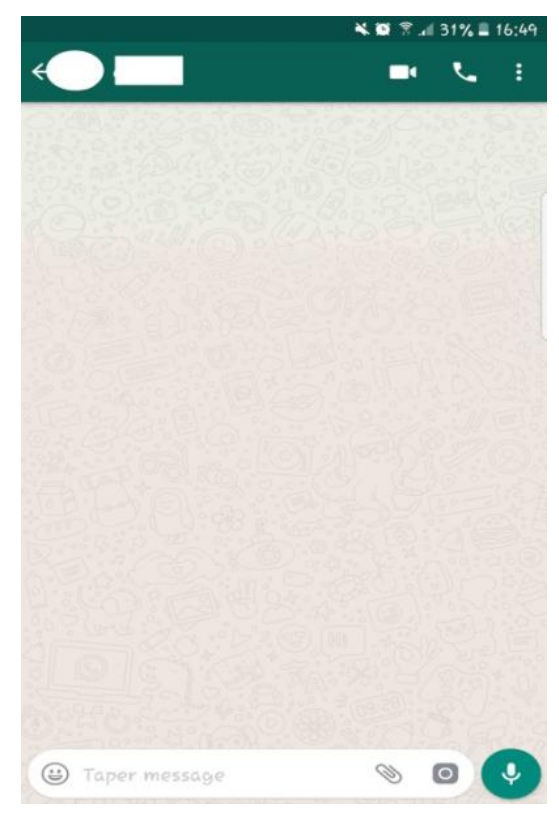

Illustration $\mathrm{n}^{\circ} 2$ : Capture d'écran du téléphone de l'un·e des auteur·e·s

Pour habiter cet espace, il faut d'abord s'y rendre puis mobiliser les affordances communicatives (Ghliss et al. à paraître). L'espace est ainsi signifiant grâce à ses éléments verbaux et ses indices sémiotiques. Par exemple, l'indication «Taper message» en bas suggère à l'usager l'action à effectuer mais aussi sa nature (le verbe d'action taper pour le message écrit). À gauche, l'émoticône indique la nature iconique que peut prendre le message si cette fonctionnalité est activée. À droite, il y a trois éléments pictographiques qui affordent des comportements communicatifs différents : le trombone (pour joindre un fichier), le carré à sa droite, censé figurer un appareil photo et indiquer à l'usager de prendre et partager en temps réel sa photo. Enfin, le cercle en vert avec le micro à l'intérieur indique la possibilité de produire des messages vocaux en instantané.

Évidemment, il ne s'agit ici que d'une représentation, les échanges WhatsApp étant, selon l'expression de Laetitia Émerit (2016), « idionumériques ${ }^{7}$ » dans le sens où ils sont dépendants de leur propre environnement numérique ${ }^{8}$. Ce que nous voulons cibler ici est l'implication de l'interface mais aussi de l'artefact connecté (smartphone) dans les interactions. Ces dernières ne sont plus l'apanage des agents humains ; elles sont pensées dans un assemblage inédit :

[...] les locutrices et les locuteurs parlent en groupe, sont mobiles dans des espaces complexes, parfois technologiquement médiatisés, et interagissent avec des humains, mais aussi avec des machines, des animaux et l'environnement en mobilisant un vaste répertoire sémiotique, verbal, gestuel, kinésique, visuel, tactile, matériel. (Greco 2018 : en ligne)

\footnotetext{
${ }^{7}$ Emerit définit les corpus «idionumériques» comme étant des corpus fortement ancrés dans leur environnement matériel et foncièrement subjectif « le compte avec lequel on accède à un contenu détermine à la fois la nature de ce contenu mais également sa présentation. Le moment durant lequel on accède à un contenu est également déterminant puisque toutes ces données sont renouvelées à chaque connexion et alimentées (à la fois en contenu mais aussi en priorité d'affichage) par les actions de l'utilisateur et des membres de son réseau » Emerit (2016 : en ligne)
} 
C'est dans cette perspective écologique du discours que nous inscrivons ce travail, et soumettons à l'analyse les discours mais aussi les actions effectuées aussi bien par les locuteurs que par l'interface WhatsApp. «C'est en cela qu'il s'agit d'une approche écologique » souligne Marie-Anne Paveau, «puisque le poste d'observation de l'analyse n'est plus le seul discours, mais l'ensemble des éléments de l'environnement. » (2017: 132)

\section{3. Habiter WhatsApp : un espace interactionnel numérique}

Afin d'examiner l'articulation de la notion d'habitat avec les espaces numériques, nous proposons d'observer trois types d'activités : 1) la constitution d'un espace interactionnel de groupe. En effet, ouvrir un groupe de discussion WhatsApp implique une coordination entre les affordances du système et les gestes des locuteurs ; 2) la référence à l'espace numérique, et pour ce faire, nous étudierons la gestion de la deixis et son alternance entre espace physique et espace numérique ; 3) enfin le travail interactionnel déployé au sein de l'application. Il s'agit donc d'une typologie exploratoire qui a pour unique but d'examiner les lieux où s'incarnent ces activités s'incarnent le mieux.

\section{1. Constitution d'un groupe WhatsApp : une dynamique technolangagière}

\section{1. 1. Ouvrir un espace, nommer un lieu}

Pour les conversations dyadiques sur WhatsApp, il suffit qu'un locuteur ou locutrice engage une discussion avec un de ses contacts pour que l'espace soit réellement effectif (voir 2. 2). Pour les conversations de groupe, la tâche exige une manipulation supplémentaire de l'usager. L'application suggère aussi de créer un groupe, le nommer et enfin sélectionner ses membres.

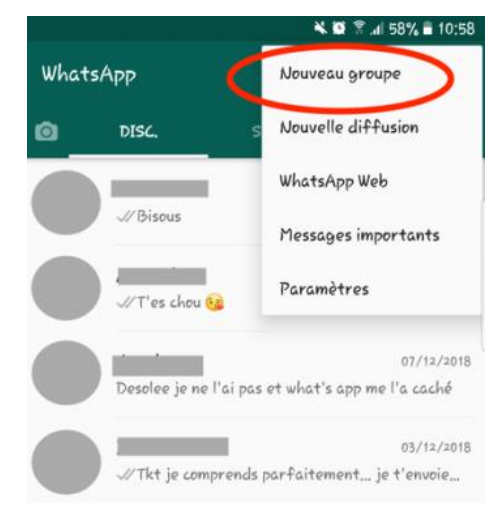

Illustration $\mathrm{n}^{\circ} 3$ : Capture d'écran depuis le téléphone de l'un·e des auteur·e·s

À partir de notre corpus, nous avons observé la dynamique de création de groupe à partir des conversations collectives. La conversation de groupe commence, comme toute première discussion entamée sur l'application, par le même message automatique de WhatsApp sur le chiffrement de bout en bout instaurant ainsi un sentiment de sécurité et de confiance auprès des usagers. Ensuite, un message supplémentaire vient s'ajouter en mentionnant que telle personne a créé le groupe, en donnant ainsi le nom du groupe comme le montre l'exemple qui suit : 


\section{Exemple ${ }^{\circ} 1:$ [Référence corpus 8b]}

À partir de cet exemple, nous pouvons identifier trois actions qui fondent la constitution de groupe. Nous avons pu observer ces mêmes phases dans tous les historiques de groupes de notre corpus. Une sorte de « pattern séquentiel systématique » (Mondada 2005) qui cadre le début de toute conversation de groupe. Tout d'abord, les actions technolangagières comme «créer un groupe » ou «ajouter un membre » dans le groupe sont instantanément décrites par l'application. Ainsi, T1, T2, T3 sont des techno-énoncés dans le sens où ils sont générés par l'algorithme de l'application qui convertit l'action de l'usager en discours verbal, une sorte de transcription hic et nunc de la manipulation. Michel $\mathrm{K}$ a donc créé un groupe qu'il a nommé «Famille », ensuite il a ajouté un de ses contacts, propriétaire de l'historique, identifié ici par le pronom «vous » dans T3 dans Michel K vous a ajouté. L'historique est donc foncièrement subjectif puisque tous les membres qui y accèdent n'auront pas par exemple la même répartition des rôles, le « vous » qui a été adressé à notre participant s'affichera chez un autre membre du groupe avec le nom de notre participant. L'interface pose ainsi le cadre de la scène d'interaction et distribue les rôles énonciatifs des membres « $\mathrm{X}$ a ajouté $\mathrm{Y}$ » et « $\mathrm{Y}$ vous a ajouté » «Vous avez ajouté $Z$ ». Il s'agit de la première phase de construction de groupe qui est celle de sélectionner les personnes qui vont faire partie du groupe. Une fois les participants sélectionnés, il faut choisir un nom pour le groupe. Nommer le groupe, c'est lui donner une identité qui permet de le distinguer des autres groupes créés. Il s'avère alors qu'à travers l'acte de nomination différentes logiques s'opèrent. Le groupe peut emprunter son nom à une catégorie préexistante comme le montre l'exemple (1) avec le nom famille. Les participants appartiennent vraisemblablement ${ }^{9}$ à la même famille et donc, pour ce cas précis, le groupe WhatsApp vient comme une extension d'un cadre relationnel hors ligne. Or, la nomination d'un groupe n'obéit pas toujours à la même logique de catégorisation comme "famille », «collègues de bureau », « voisins du quartier », etc. Elle peut aussi être motivée par le choix d'un objectif justifiant ainsi la légitimité du groupe comme l'illustre cet exemple :

$\mathrm{T} 1$

$\mathrm{T} 2$
18/06/2016, 00:42 - Les messages que vous envoyez dans ce groupe sont désormais protégés avec le chiffrement de bout en bout. Appuyez pour plus d'informations.

18/06/2016, 00:42 - Vous avez créé le groupe « Cadeau Michel »

Exemple $n^{\circ} 2:$ [Référence corpus A]

Dans cet extrait, nous retrouvons les mêmes membres de la famille précédente à l'exception de Michel. Le nom «Cadeau Michel » laisse donc entendre que les membres de la famille discutent en cachette pour décider du choix du cadeau. La conversation se poursuit comme suit :

$\mathrm{T} 3$

$\mathrm{T} 4$

T5
18/06/2016, 00:43 - Robin K : Vous voulez vraiment prendre un iPhone à Michel ? :o

18/06/2016, 00:43 - Robin K: Un casque je pense qu'il a une idée assez précise de ce qu'il veut donc vaut mieux qu'il l'achète lui même

18/06/2016, 07:36 - Maman: ben je sais pas faut rester dans lé budget le casque ca pourrais le faire et cs ière pour dimanche

\footnotetext{
${ }^{9}$ Pour des raisons éthiques et afin de préserver l'anonymat de nos participants, nous avons changé tous les prénoms. Or pour cet exemple en particulier tous les membres avaient le même nom de famille dont nous gardons que la lettre initiale $\mathrm{K}$.
} 
La création d'un groupe WhatsApp avec les mêmes locuteurs et locutrices à l'exception d'un, conforte l'hypothèse de la discussion en cercle restreint ${ }^{10}$. Les actions d'inclusion et l'exclusion des membres sont constitutives de l'identité du groupe de discussion. La création du groupe est ici corrélée à la réalisation d'un objectif commun. Le groupe WhatsApp se présente comme un cadre interactionnel, un espace de l'entre-soi pour reprendre Bachelard, mais aussi un espace clos protégé de toute intrusion et inférence extérieures aidant ainsi à l'accomplissement de l'objectif. De même que T1, T7 est aussi un techno-énoncé généré par l'application. Le locuteur, enregistré en tant que «Papa » par notre participant, a quitté le groupe de discussion. WhatsApp convertit alors cette action en message verbal ce qui donne «Papa est parti » en T7, enregistré un mois après la séquence interactionnelle. Ce tour est aussi le dernier tour enregistré dans cet historique de conversation et vient ainsi marquer la clôture de l'interaction globale.

Les propriétés objectifs de l'espace (ses affordances, ses prises) servent ainsi à créer un environnement, soit un monde qui fait sens pour un groupe d'individus et qui prend forme à partir des buts qu'ils se fixent, comme l'avait bien vu Ingold (voir plus haut). L'espace ainsi créé est intrinsèquement lié à ses buts : c'est la raison pour laquelle il est en partie délaissé, une fois qu'ils sont atteints.

\section{1. 2. Séquence d'ouverture ou l'invitation à cohabiter}

La création du groupe à elle seule ne suffit pas pour dire l'habiter ; c'est l'activité interactionnelle incarnée dans et par cet espace numérique qui fonde l'espace de socialisation. Un des participants vient amorcer la conversation rendant ainsi l'espace habitable. On reprendra ici les deux exemples précédents pour observer la dynamique discursive déployée à l'ouverture de chaque séquence. Pour l'exemple $\mathrm{n}^{\circ} 2$, Robin $\mathrm{K}$ ouvre la séquence en $\mathrm{T} 3$ : «Vous voulez vraiment prendre un iPhone à Michel ? :o » et poursuit en T4: «Un casque je pense qu'il a une idée assez précise de ce qu'il veut donc vaut mieux qu'il l'achète lui même ». La locutrice «maman » répond en T5: «ben je sais pas faut rester dans lé budget le casque ca pourrais le faire et cs ière pour dimanche » et en T6: «sa urge ».

La mobilisation de ressources langagières. Pour l'exemple (1) groupe, Michel K écrit en T4 « Plop. Vous faites quoi cet après midi ? ». L'interjection plop est une onomatopée utilisée à la base dans les messageries instantanées comme une salutation. L'interrogation qui s'ensuit a une valeur purement phatique. Les participants ne répondent pas à l'interrogation et ne donnent donc pas suite à la conversation. Le groupe reste inerte et aucun échange ne se déroule pendant plus de deux semaines jusqu'à la nouvelle relance de Michel K :

\footnotetext{
${ }^{10}$ Restreint par rapport au groupe préexistant notamment.
} 
17/07/2016, 10:29 - Les messages que vous envoyez dans ce groupe sont désormais protégés avec le chiffrement de bout en bout. Appuyez pour plus d'informations.

\begin{tabular}{|c|c|}
\hline T2 & 17/07/2016, 09:39 - Michel K a créé le groupe "Famille" \\
\hline T3 & 17/07/2016, 10:29 - Michel K vous a ajouté \\
\hline T4 & 17/07/2016, 09:39 - Michel K : Plop. Vous faites quoi cet après midi ? \\
\hline T5 & 03/08/2016, $20: 18$ - Michel K : <Fichier omis> \\
\hline T6 & 03/08/2016, 20 :41 - Robin K : Trop drôle ! \\
\hline T7 & 03/08/2016, $20: 41$ - Robin $\mathrm{K}$ : T'as fait l'école du rire? \\
\hline T8 & 03/08/2016, $20: 42$ - Robin $\mathrm{K}:$ T'as mangé un clown ? \\
\hline T9 & 03/08/2016, $20: 51$ - Maman : elle a I air trop bonne pour toi tout seul ? \\
\hline T10 & 03/08/2016, $20: 52$ - Maman : ben il a raison faut mettre des gants \\
\hline T11 & $\begin{array}{l}\text { 03/08/2016, } 21: 10 \text { - Robin } \mathrm{K} \text { : Non mais avoue t'as rit Oui pour moi. On allait } \\
\text { perdre les pêches sinon. }\end{array}$ \\
\hline T12 & 03/08/2016, $21: 12$ - Maman : moi j ai pas rigolé \\
\hline T13 & 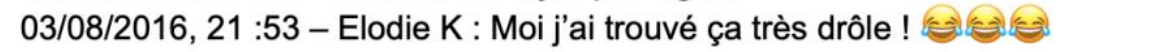 \\
\hline
\end{tabular}

\section{Exemple $\mathrm{n}^{\circ} 1$ bis [Référence corpus $8 \mathrm{~B}$ ]}

La relance de Michel K en T5 avec l'envoi d'un fichier image, a priori à caractère humoristique, a suffi à déclencher les réactions des autres membres et par conséquent leur participation à l'échange dans le groupe de discussion. La séquence qui suit décrit la scène de rire collective : l'exclamation formulée par Robin «Trop drôle ! en T6 suivie de deux interrogations «T'as fait l'école du rire? T'as mangé un clown ? » en T7 et T8. Ensuite, l'expression du rire (T11 et T13 ?) et de l'amusement se poursuit entre partage («Moi j'ai trouvé ça très drôle !» (en T12 «moi j ai pas rigolé »). Par ailleurs, on notera que le rire se déploie différemment d'un tour à un autre ; s'il est thématisé en (T11, T12), on le trouve clairement exprimé et sémiotisé par le choix d'émoji en fou-rire (visage qui sourit avec les larmes de joie).

L'observation microscopique de la mise en place des groupes WhatsApp révèle deux points fondamentaux : en premier lieu, l'activité interactionnelle est rendue possible grâce à la mobilisation des compétences technolangagières telles que la création de groupe, la sélection et l'ajout des participants et le partage de fichiers iconique. En second lieu, il y a un savoir-faire social et langagier à maîtriser comme l'amorce de la conversation de groupe une fois l'espace créé ou la connaissance des lexiques comme plop, les onomatopées comme hahah et les émojis.

\section{2. La référence de l'espace numérique : la gestion de la deixis}

Si la description déictique de l'espace est un thème classique de la linguistique de la référence spatiale (Mondada 2005), notre propos ici est d'observer l'organisation déictique dans un contexte mêlant espace en ligne et hors ligne comme le montre cet exemple :

$\begin{array}{ll}\text { T1 } & \text { 29/04/2017, 13:31 - Hana }: \text { T la } \\ \text { T2 } & \text { 29/04/2017, 13:31 - Sana }: \text { Cc oui } \\ \text { T3 } & \text { 29/04/2017, 13:31 - Sana: T où } ? \\ \text { T4 } & \text { 29/04/2017, 13:32 - Hana: Café de France } \\ \text { T5 } & \text { 29/04/2017, 13:32 - Sana }: \text { J arrive } \\ \text { T6 } & \text { 29/04/2017, 13:33 - Hana: Ok r loin } ? \\ \text { T7 } & \text { 29/04/2017, 13:41 - Sana: Non }\end{array}$


Pour cet extrait, deux amies se retrouvent pour un voyage au Maroc. La séquence interactionnelle présentée ici donne lieu à un jeu espace-temps très intéressant: Hana ouvre l'échange avec un T1 : «T la». Malgré l'absence de toute marque interrogative, la contextualisation de l'énoncé nous fait facilement comprendre qu'il s'agit ici d'une interrogation d'autant plus que son amie Sana répond aussitôt par «oui » en $\mathrm{T} 2$. Le déictique spatial là dans « $\mathrm{T}$ là » renvoie à l'espace numérique WhatsApp. Hana demande en effet si son amie est connectée ou encore en ligne. Une fois que les deux amies sont présentes simultanément, l'échange se construit comme suit. Sana pose à son tour une question : «T où ? » en T3, son amie répond : «Café de France » et donne ainsi sa localisation dans l'espace physique. Hana demande à son tour la location de son amie pour pouvoir la situer par rapport à l'endroit où elle même se trouve : «t'es loin ?» en T6. Sana répond : «non » (T7). Les tours T8, T9 ET T10 renvoient à une anecdote - discours enchâssé - qui concentre un interdiscours échappant à la conversation.

Ce qui est important à relever dans cet échange est le jeu des déictiques spatiaux qui se déploient à un double niveau. La référence spatiale jongle entre l'espace numérique en T1 avec l'adverbe là et l'espace physique en T3 avec l'adverbe où. Les locutrices investissent les mêmes ressources linguistiques dans la gestion des deux espaces différents à savoir les espaces hors ligne et en ligne. Pour autant, ces deux derniers investissent dans le même espace interactionnel. Si WhatsApp est ici actualisé dans la scène énonciative, l'énoncé « $\mathrm{J}$ arrive » en T5 laisse à entendre que l'espace physique prend en charge la conversation lors de la rencontre.

Les espaces en ligne et hors ligne se superposent pour composer simultanément l'espace interactionnel. Ce dernier, qui se trouve ici à l'intersection des sphères physique et numérique, est souvent organisé à partir des actions des locuteurs, sa gestion ou comme le précise Mondada, sa description «est organisée de manière interactionnelle, en s'ajustant aux circonstances de son énonciation, négociation et élaboration collective » (Mondada 2005 : 7). Par conséquent, il n'est plus possible de les opposer : elles évoluent dans un espace hybride, comme en avaient fait l'hypothèse des chercheurs comme Doueihi (2011), Paveau (2012) et (2017), Greco (2018).

\section{3. L'espace WhatsApp, cadre des activités sociales transformées?}

L'observation du corpus a montré une panoplie de séquences interactionnelles mettant en scène des activités sociales de la vie quotidienne : anniversaire, conversation autour d'un film, concertation pour organiser un évènement, etc. Dans l'exemple qui suit, l'activité dont il est question est un apéro entre amis : 


\begin{tabular}{|c|c|}
\hline T1 & 04/02/2016 21:30:41: Julien H: Apéro? \\
\hline T2 & 04/02/2016 21:30:47: Ives : Exacte hajaha \\
\hline T3 & 04/02/2016 21:31:00: Julien H: Moi c'est pour bientôt hahaha \\
\hline T4 & 04/02/2016 21:31:02: Ives : J allais te dire (2) $: \cdot$ \\
\hline T5 & 04/02/2016 21:31:33: Julien H: A coup de binouses ou bien? \\
\hline T6 & 04/02/2016 21:31:35: Ives : <image absente> \\
\hline $\mathrm{T} 7$ & 04/02/2016 21:31:45: Julien H: Aaaaah \\
\hline T8 & 04/02/2016 21:31:48: Julien H: Le bon rhum \\
\hline T9 & 04/02/2016 21:32:06: Ives : Troooooo \\
\hline T10 & 04/02/2016 21:32:23: Julien H: Cuba libre ? \\
\hline T11 & 04/02/2016 21:33:25: Ives : Exacte \\
\hline $\mathrm{T} 12$ & 04/02/2016 21:33:46: Julien H: Putain ça donne envie \\
\hline T13 & 04/02/2016 21:34:32: Ives : Arret !! On c est mis bien quand meme! \\
\hline T14 & 04/02/2016 21:35:29: Julien H: Ouais hahaha \\
\hline T15 & 04/02/2016 21:48:10:Ives: \& ouai ien garderai un PUTIN de souvenir \\
\hline T16 & 04/02/2016 22:45:00: Julien H: Putain ouais je suis d'accord! \\
\hline
\end{tabular}

Exemple 4 [Référence corpus 7b]

L'extrait est tiré d'un historique de discussion entre deux amis habitant deux pays différents (la Belgique pour l'un et la France pour l'autre). À première vue, l'extrait présente une conversation on ne peut plus banale entre deux amis, conversation teintée d'humour et d'amusement. Mais si l'on examine de plus près ce qui se joue à travers cet extrait on identifiera aisément un «jeu », un apéro basculé dans un nouveau cadre. Pour expliquer au mieux ce phénomène, on mobilisera la notion de «cadre» de Goffman (1991) et de «jeu» chez Bateson (1955). Ce dernier, ayant observé le comportement ludique chez les loutres, soulignait que les animaux pouvaient distinguer le vrai combat du faux. Goffman s'est donc appuyé sur cette observation pour indiquer qu'il existe différents cadres de l'expérience, le «cadre primaire » - à savoir pour le cas des loutres, le combat - et le « cadre secondaire » qui serait ici le jeu simulant le schéma d'action du premier pour y introduire des altérations.

Certains éléments de ce schème seront pourtant systématiquement altérés : par exemple, si les loutres jouent à se mordre, aucune morsure n'est sérieuse. En somme, on a là une transcription, une transposition, une transformation, c'est le terme que nous retiendrons $[\ldots]$ au sens de la géométrie : une séquence de jeu se substitue à une séquence de combat. (Goffman 1991 : 49)

Pour notre exemple, ce n'est pas un combat que les locuteurs essaient de «jouer » mais un moment convivial : la séquence s'ouvre en T1 avec l'interrogation elliptique : «Apéro ? » que l'interlocuteur confirme en ponctuant avec un rire « hahaha ». Julien répond à son tour : « moi c'est pour bientôt » et ponctue lui aussi sa phrase par un rire. La répétition du geste du rire ici renvoie au phénomène d'échoïsation empathique ${ }^{11}$ (voir Cosnier \& Brunel 1997; Cosnier \& Huyghues-Despointes 2000) et participe au travail de coordination observé généralement dans les interactions orales où l'on reproduit d'une manière plus ou moins inconsciente la posture et les mimiques de son interlocuteur pour souligner son cophasage. La séquence présente un cadre transformé de l'apéro. Ce dernier se définit

${ }^{11}$ L'échoïsation empathique (Cosnier \& Huyghues-Despointes 2000) est définie comme étant l'identification de l'état affectif apparenté du locuteur et sa reproduction par l'interlocuteur. Le phénomène est généralement trop discret pour être perceptible par un observateur, il est par conséquent qualifié de «sublimiaine » mais peut être identifié par quelques manifestations comme des sourires simultanés, analogies mimiques, changement en miroir de positions corporelles, etc. 
comme un moment de partage et socialisation; il se voit ici pris dans une nouvelle dynamique et transposé dans un espace numérique. Ainsi, les locuteurs reproduisent les actions effectuées dans un « vrai » apéro (conversation autour du choix de la boisson par exemple) mais ces actions se trouvent altérées, dans la mesure où, comme les loutres qui jouent à se mordre pour de faux, les locuteurs ici trinquent - action simulée avec les émojis (en T14) - et claquent des mains (en T15) pour de faux. Dans une approche conversationnelle, on souligne aisément la dynamique de coordination des gestes, de synchronisation et enfin d'échoïsation mobilisée en vue de garder le caractère décontracté et fluide de la conversation ordinaire lors d'un apéro. Ainsi, les signes de WhatsApp se voient dotés de nouvelles possibilités ou affordances que découvrent les locuteurs à partir des buts qu'ils se fixent implicitement (consolider leur relation amicale).

\section{Conclusion}

Dans cet article, l'idée était de vérifier l'hypothèse de l'habitat des espaces numériques. Pour ce faire nous avons montré comment l'interaction prend place dans un lieu comme WhatsApp et comment les locuteurs investissent et exploitent les affordances de cet espace pour y habiter. L'analyse s'est focalisée sur trois points fondamentaux : En premier lieu, la conception de l'espace de discussion. En effet, la conception de Mondada de «l'espace (...) comme une ressource pour l'action, réflexivement contrainte et configurée par elle » (2005: 7) est attestée avec l'application numérique WhatsApp qui, à partir de ses affordances techniques, propose certes un espace de discussion mais organise et planifie également les différentes actions effectuées. L'analyse discursive de la création de groupe de WhatsApp a montré que cette action est coordonnée simultanément par les locuteurs et par l'application mobile. En deuxième lieu, et dans la mesure où nous assistons à une reconfiguration de l'espace interactionnel, nous nous sommes intéressés à la référence spatiale dans un environnement complexe où se superposent les deux espaces en ligne et hors ligne. Dans cette optique, l'intérêt était alors d'observer la redistribution de la deixis et des ressources linguistiques par les locuteurs qui interagissent d'une manière indifférenciée dans les deux espaces. Enfin, en mobilisant notamment la notion de cadre de Goffman (1991), nous avons montré comment les locuteurs habitent l'espace WhatsApp en y reproduisant des activités sociales. Ces mêmes activités, se trouvent alors transposées dans un nouveau cadre qui les convertit dans l'espace numérique et les réorganise selon ses propres modalités sémiotiques.

\section{Références bibliographiques}

Agostini-Marchese E. (2017), «Les structures spatiales de l'éditorialisation. Terre et mer de Carl Schmitt et l'espace numérique », Sens public. En ligne : 〈http://sens-public.org/article1238.html>, consulté le 11/01/2019.

Bachelard G. (1957), Poétique de l'espace, Paris, PUF.

Bateson G. (1955), «A theory of play and fantasy; a report on theoretical aspects of the project of study of the role of the paradoxes of abstraction in communication », Psychiatric research reports 2, p. 39-51.

Cosnier J. \& Brunel M.-L. (1997), « De l'interactionnel à l'intersubjectif », dans Marcarino A. (dir.) (1997), Analisi delle conversazione e prospettive di ricerca in etnometodologia, Urbino, QuattroVenti, p. 151-163. 
Cosnier J. \& Huyghues-Despointes S. (2000), «Les mimiques du créateur, ou l'autoréférence des représentations affectives », Collection éthologie et psychologie des communications, p. 157-168.

Doueihi M. (2011), Pour un humanisme numérique, Paris, Seuil.

Émerit L. (2016), «La notion de lieu de corpus : un nouvel outil pour l'étude des terrains numériques en linguistique », Corela 14(1), mis en ligne le 16/06/2016, consulté le 01/03/2019. En ligne: $<$ https://journals.openedition.org/corela/4594>.

Ghliss Y. (à paraître en 2019), «Les photo-discours via WhatsApp: de l'affordance mobile à l'agentivité affective», Corela (dossier Les affordances langagières: textualité numérique, matérialité discursive).

Ghliss Y., Perea F. \& Ruchon C. (dir.) (à paraître en 2019), «Les affordances langagières : textualité numérique, matérialité discursive », Corela.

Gibson J. J. (1977), «The theory of affordances », dans Shaw R. \& Brandsford J. (dir.), Perceiving, Acting, and Knowing: Toward and Ecological Psychology,New York, John Wiley \& Sons Inc, p. 6282.

Goffman E. (1991), Les Cadres de l'expérience, Paris, Éditions de Minuit.

Greco L. (2018), «L'interaction au prisme de l'intercorporéité : repenser les relations entre langage, cognition et culture », Langage \& Société 165(3), p. 169-177.

Herouard F. (2007), « Habiter et espace vécu : une approche transversale pour une géographie de l'habiter », dans Paquot T., Lussault M . \& Younès C. (dir.) (2007), Habiter, le propre de l'humain. Villes, territoires et philosophie, Paris, La Découverte, p. 159-170.

Ingold T. (2013), Marcher avec les dragons, Paris, Zones Sensibles Éditions.

Kitchin R. \& Dodge M. (2011), Code/Space, Software and Everyday Life, Cambridge, MIT Press.

Leroi-Gourhan A. (1945), Évolution et techniques. Milieux et techniques, Paris, Albin Michel, tome 2.

Mondada L. (2005), «La constitution de l'origo déictique comme travail interactionnel des participants : une approche praxéologique de la spatialité », Intellectica 2(3), p. 41-42.

Mondada L. (2012), «Espaces en interaction : espace décrit, espace inscrit et espace interactionnel, dans un débat d'urbanisme participatif » dans Bulletin VALS-ASLA, p. 15-42.

O’Hara K. P., Massimi M., Harper R., Rubens S. \& Morris J. (2014), «Everyday dwelling with WhatsApp », dans Proceedings of the 17th ACM conference on Computer supported cooperative work \& social computing, ACM, p. 1131-1143.

Paquot T., Lussault M . \& Younès C. (dir.) (2007), Habiter, le propre de l'humain. Villes, territoires et philosophie, Paris, La Découverte, p. 103-125.

Paveau M.-A. (2012), «Ce que disent les objets. Sens, affordance, cognition», Synergies Pays riverains de la Baltique 9, p. 53-65. 
Paveau M.-A. (2013), «Technodiscursivités natives sur Twitter. Une écologie du discours numérique », Épistémé (Revue internationale de sciences humaines et sociales appliquées, Séoul) 9, p. $139-176$.

Paveau M.-A. (2017), L'analyse du discours numérique. Dictionnaire des formes et des pratiques, Paris, Hermann.

Stock M. (2007), « Théorie de l'habiter. Questionnements » dans Paquot T., Lussault M. \& Younès C. (dir.) (2007), Habiter, le propre de l'humain. Villes, territoires et philosophie, Paris, La Découverte, p. 103-125. 